\title{
Computational Analysis of Complex Population Dynamical Model with Arbitrary Order
}

\author{
Fazal Haq ${ }^{D}$, ${ }^{1}$ Kamal Shah $\left(\mathbb{D},{ }^{2}\right.$ Ghaus ur Rahman, ${ }^{3}$ \\ Yongjin $\mathrm{Li}\left(\mathbb{D},{ }^{4}\right.$ and Muhammad Shahzad $\left(\mathbb{D}^{1}\right.$ \\ ${ }^{1}$ Department of Mathematics, Hazara University Mansehra, Khyber Pakhtunkhwa, Pakistan \\ ${ }^{2}$ Department of Mathematics, University of Malakand, Dir(L), Khyber Pakhtunkhwa, Pakistan \\ ${ }^{3}$ Department of Mathematics and Statistics, University of Swat, Mingora, Pakistan \\ ${ }^{4}$ Department of Mathematics, Sun Yat-sen University, Guangzhou, China
}

Correspondence should be addressed to Yongjin Li; stslyj@mail.sysu.edu.cn

Received 26 September 2017; Accepted 10 December 2017; Published 3 January 2018

Academic Editor: Jesus Medina

Copyright (C) 2018 Fazal Haq et al. This is an open access article distributed under the Creative Commons Attribution License, which permits unrestricted use, distribution, and reproduction in any medium, provided the original work is properly cited.

\begin{abstract}
This paper considers the approximation of solution for a fractional order biological population model. The fractional derivative is considered in the Caputo sense. By using Laplace Adomian decomposition method (LADM), we construct a base function and provide deformation equation of higher order in a simple equation. The considered scheme gives us a solution in the form of rapidly convergent infinite series. Some examples are used to show the efficiency of the method. The results show that LADM is efficient and accurate for solving such types of nonlinear problems.
\end{abstract}

\section{Introduction}

Nowadays in most of the research areas, the importance of fractional differential equations has been increased due to its wide range of applications in real world problems. In different scientific and engineering categories such as chemistry, mechanics, and physics applications of fractional calculus can be found. It can be also used in control theory, optimization theory, image processing, economics, and so on [1-5]. Mathematical models of fractional order are very important to study natural problems. As is known, the nature of the trajectory of the fractional order derivatives is nonlocal, describing that the fractional order derivative possesses memory effect features and any dynamical or physical system related to fractional order differential operators has a memory effect, which shows that the future states depend on the present and past states. The limitations of Caputo fractional derivative are removed while introducing Caputo-Fabrizio derivative; see [6]. This new approach was used by Singh et al. to model the dynamics of computer viruses, particularly in those cases where the physical processes do not bear plasticity, fatigue, damage, and electromagnetic hysteresis effects. In view of the great significance of new fractional derivative, a fractional order model of chemical kinetic system with full memory effect has been studied very well. The authors examined the existence and uniqueness of the solutions for chemical kinetic system of arbitrary order by using the fixed-point theorem.

System of nonlinear differential equations are very important for mathematicians, engineers, and physicists, because in most of physical systems the input is not proportional to output in nature. In addition to the study of simple nonlinear differential equations, exact solution of the nonlinear evolution equation also plays an important role in the study of some nonlinear problems. For the exact solution there are many approaches such as Hirota's method, Darboux transformation, and Painleve expansions.

In last few years, more alternative numerical methods have been used for solving both linear and nonlinear problems of physical interest including homotopy perturbation method (HPM) [7, 8], Adomian decomposition method $(\mathrm{ADM})[9,10]$, and homotopy analysis method (HAM) [11]. Due to various applications of Lienard's equation, Kumar et al. proposed a numerical algorithm based on fractional 
homotopy analysis transform method to study fractional order Lienard's equation, while recently in 2017 the authors used $q$-homotopy analysis method and Laplace transform approach to explore some aspects of the FitzHugh-Nagumo equation of fractional order. Laplace decomposition method was adopted in $[12,13]$ to deal with some other nonlinear problems while homotopy perturbation transform method is selected in [14] to explore the approximate solution. Very recently Laplace transform combined with homotopy analysis method is used to produce effective method called homotopy analysis transform method (HATM) [15, 16]. Its main purpose is to handle nonlinear physical problems. Baleanu et al. [17] have solved the fractional order optimal control problems. For the solution of system of SchrodingerKorteweg-de Vries equation, Golmankhaneh has used the homotopy perturbation method [18]. In [19], the authors presented the following nonlinear fractional order biological population model using homotopy analysis transform method:

$$
D_{t}^{\alpha} v(w, z, t)=\left(D_{x}^{2}+D_{z}^{2}\right) v^{2}(w, z, t)+f(v(w, z, t)),
$$

with initial condition as

$$
v(w, z, 0)=f_{0}(w, z),
$$

where $\partial / \partial t=D_{t}, \partial^{2} / \partial w^{2}=D_{w}^{2}$, and $\partial^{2} / \partial z^{2}=D_{z}^{2}$. Further, $v$ represents density of the population and $f$ denotes supply of the population due to birth and death rate. This nonlinear fractional order biological population model is obtained by replacing the first-order derivative term in the corresponding biological population model by fractional order $\alpha$, where $0<\alpha \leq 1$. The derivative is considered in Caputo's sense. The parameter involved in fractional derivative shows various responses. If $\alpha=1$, the fractional order biological model reduces to standard biological model. In [20, 21], the authors discussed such models while exploring their different aspects. Promoting the above work, we solve model (1) by using LADM.

$$
D_{t}^{\alpha} v=\left(D_{w}^{2}+D_{z}^{2}\right) v^{2}-k r v^{(a+b)}+k v^{a}
$$

with the given initial condition

$$
v(w, z, 0)=f_{0}(w, z) .
$$

Since the proposed model is highly nonlinear and contains nonlinear terms $v^{2}$ and $v^{(a+b)}$ for $a+b \neq 1$, therefore, we decompose $v^{2}$ and $v^{(a+b)}$ in terms of Adomian polynomials as

$$
\begin{aligned}
v(w, z, t) & =\sum_{m=0}^{\infty} v_{m}(w, z, t), \\
v^{a}(w, z, t) & =\sum_{m=0}^{\infty} T_{m}, \\
v^{2}(w, z, t) & =\sum_{m=0}^{\infty} P_{m}, \\
v^{a+b}(w, z, t) & =\sum_{m=0}^{\infty} Q_{m},
\end{aligned}
$$

where

$$
\begin{aligned}
& T_{m}=\left.\frac{1}{n !} \frac{d^{n}}{d \lambda^{n}}\left[\sum_{i=0}^{\infty}\left(\lambda^{i} v_{i}\right)^{a}\right]\right|_{\lambda=0}, \\
& P_{n}=\left.\frac{1}{n !} \frac{d^{n}}{d \lambda^{n}}\left[\sum_{i=0}^{\infty}\left(\lambda^{i} v_{i}\right)^{2}\right]\right|_{\lambda=0}, \\
& Q_{n}=\left.\frac{1}{n !} \frac{d^{n}}{d \lambda^{n}}\left[\sum_{i=0}^{\infty}\left(\lambda^{i} v_{i}\right)^{a+b}\right]\right|_{\lambda=0} .
\end{aligned}
$$

\section{Preliminaries}

In order to assist the readers, here in this section we recall some fundamental definitions and results from fractional calculus.

Definition 1 (see [22]). The fractional order derivative of $f(x)$ in the Caputo sense is defined as

$$
\begin{aligned}
{ }^{c} D^{\alpha} f(x) & =I^{n-\alpha}\left[{ }^{c} D^{n} f(x)\right] \\
& =\frac{1}{\Gamma(n-\alpha)} \int_{0}^{x}(x-\tau)^{n-\alpha-1} f^{n}(\tau) d \tau .
\end{aligned}
$$

For $n-1 \leq \alpha \leq n, n \in N$. In case of function $f(x, t)$, we defined Caputo derivative with respect to " $x$ " as

$$
\begin{aligned}
{ }^{c} D^{\alpha} f(x, t) & =I^{n-\alpha} \frac{\partial^{n}}{\partial x^{n}} f(x, t) \\
& =\frac{1}{\Gamma(n-\alpha)} \int_{0}^{x}(x-\tau)^{n-\alpha-1} \frac{\partial^{n}}{\partial \tau^{n}} f(\tau, t) d \tau .
\end{aligned}
$$

Further for $f(t)=t^{\delta}$, we have ${ }^{c} D^{\alpha}\left[t^{\delta}\right]=\Gamma(\delta+1) t^{\delta-\alpha} / \Gamma(\delta+$ $1-\alpha)$.

Definition 2 (see [22]). The Riemann-Liouville fractional order integral operator of order $\alpha>0$ of function $f(x)$ is given as

$$
\begin{aligned}
& I^{\alpha} f(x)=\frac{1}{\Gamma(\alpha)} \int_{0}^{x}(x-\tau)^{\alpha-1} f(\tau) d \tau . \\
& I^{0} f(x)=f(x) .
\end{aligned}
$$

In case of $f(x, t)$ then the Riemann-Liouville fractional order integral operator of order $\alpha>0$ is given by

$$
\begin{aligned}
& I^{\alpha} f(x, t)=\frac{1}{\Gamma(\alpha)} \int_{0}^{x}(x-\tau)^{\alpha-1} f(\tau, t) d \tau . \\
& I^{0} f(x, t)=f(x, t) .
\end{aligned}
$$

The Riemann-Liouville fractional order integral [22] is given by

$$
I^{\alpha}\left[t^{\gamma}\right]=\frac{\Gamma(\gamma+\alpha)}{\Gamma(\gamma+\alpha+1)} t^{\alpha+\gamma},
$$

provided that integral on the right side is point wise defined on $(0, \infty)$. 
Lemma 3. Let $0<\alpha \leq 1$ and $\Psi \in L^{1}(0,1)$. Then

$$
D^{\alpha} I^{\alpha} \Psi(t)=\Psi(t)
$$

hold.

$$
I^{\alpha}\left[{ }^{c} D^{\alpha} \Psi(t)\right]=\Psi(t)-\frac{\left[{ }^{c} D^{\alpha-1} \Psi(t)\right]_{t=0}}{\Gamma(\alpha)} t^{\alpha-1}
$$

holds almost everywhere on $(0,1)$.

Definition 4 (see [19]). The Laplace transform of the Caputo derivative is given as

$$
\begin{aligned}
& \mathscr{L}\left\{{ }^{c} D^{\alpha} y(t)\right\}=s^{\alpha} Y(s)-\sum_{k=0}^{n-1} s^{\alpha-k-1} y^{(k)}(0), \\
& n-1<\alpha<n, n \in N .
\end{aligned}
$$

Definition 5 (see [23]). The Mittag-Leffler function in term of power series is defined as

$$
E_{\alpha}(t)=\sum_{k=0}^{\infty} \frac{t^{k} \alpha}{\Gamma(k \alpha+1)}, \quad t \in \mathscr{C}, \operatorname{Re}(\alpha)>0
$$

\section{LADM for Biological Model}

This section is devoted to the general procedure of the LADM for solving (3) with given initial conditions. To apply Laplace transform to model (3) we proceed as

$$
\begin{aligned}
\mathscr{L}\left\{D_{t}^{\alpha} v\right\}=\mathscr{L}\left\{\left(D_{w}^{2}+D_{z}^{2}\right) v^{2}-k r v^{(a+b)}+k v^{a}\right\}, \\
t>0, w, z \in R, 0<\alpha \leq 1,
\end{aligned}
$$

with the given initial condition

$$
v(w, z, 0)=f_{0}(w, z)
$$

From definition of Laplace transform on both sides of (3), we have

$$
\begin{aligned}
s^{\alpha} \mathscr{L} & \{v(w, z, t)\}-s^{\alpha-1} v(w, z, 0) \\
& =\mathscr{L}\left\{\left(D_{w}^{2}+D_{z}^{2}\right) v^{2}-k r v^{(a+b)}+k v^{a}\right\} .
\end{aligned}
$$

Using given initial conditions yields that

$$
\begin{aligned}
\mathscr{L}\{v(w, z, t)\} \\
=\frac{1}{s} f_{0}(w, z) \\
\quad+\frac{1}{s^{\alpha}} \mathscr{L}\left\{\left(D_{w}^{2}+D_{z}^{2}\right) v^{2}-k r v^{(a+b)}+k v^{a}\right\} .
\end{aligned}
$$

Therefore, after decomposing nonlinear terms in terms of Adomian polynomials and considering the unknown solutions $v=\sum_{n=0}^{\infty} v_{n},(18)$ can be written as

$$
\begin{aligned}
\mathscr{L} & \left\{\sum_{n=0}^{\infty} u(x, y, t)\right\} \\
= & \frac{1}{s} f_{0}(w, z) \\
& +\frac{1}{s^{\alpha}} \mathscr{L}\left\{\left(D_{w}^{2}+D_{z}^{2}\right) \sum_{n=0}^{\infty} P_{n}-k r \sum_{n=0}^{\infty} Q_{n}+k \sum_{n=0}^{\infty} T_{n}\right\} .
\end{aligned}
$$

Comparing terms on both sides, we get

$$
\begin{aligned}
& \mathscr{L}\left\{v_{0}(w, z, t)\right\}=\frac{1}{s} f_{0}(w, z), \\
& \mathscr{L}\left\{v_{1}(w, z, t)\right\} \\
& \quad=\frac{1}{s^{\alpha}} \mathscr{L}\left\{\left(D_{w}^{2}+D_{z}^{2}\right) P_{0}-k r Q_{0}+k v_{0}\right\}, \\
& \mathscr{L}\left\{v_{2}(w, z, t)\right\} \\
& \quad=\frac{1}{s^{\alpha}} \mathscr{L}\left\{\left(D_{w}^{2}+D_{z}^{2}\right) P_{1}-k r Q_{1}+k v_{1}\right\}, \\
& \mathscr{L}\left\{v_{3}(w, z, t)\right\}=\frac{1}{s^{\alpha}} \mathscr{L}\left\{\left(D_{w}^{2}+D_{z}^{2}\right) P_{2}-k r Q_{2}+k v_{2}\right\}
\end{aligned}
$$

$$
\begin{aligned}
\mathscr{L} & \left\{v_{n+1}(w, z, t)\right\} \\
& =\frac{1}{s^{\alpha}} \mathscr{L}\left\{\left(D_{w}^{2}+D_{z}^{2}\right) P_{n}-k r Q_{n}+k v_{n}\right\}, \quad n \geq 1 .
\end{aligned}
$$

After taking Laplace inverse transform of system (21), we get

$$
\begin{aligned}
& v_{0}(w, z, t)=f_{0}(w, z), \\
& \begin{array}{l}
v_{1}(w, z, t) \\
=\mathscr{L}^{-1}\left[\frac{1}{s^{\alpha}} \mathscr{L}\left\{\left(D_{w}^{2}+D_{z}^{2}\right) P_{0}-k r Q_{0}+k v_{0}\right\}\right], \\
v_{2}(w, z, t) \\
\quad=\mathscr{L}^{-1}\left[\frac{1}{s^{\alpha}} \mathscr{L}\left\{\left(D_{w}^{2}+D_{z}^{2}\right) P_{1}-k r Q_{1}+k v_{1}\right\}\right], \\
\quad \vdots \\
v_{n+1}(w, z, t) \\
=\mathscr{L}^{-1}\left[\frac{1}{s^{\alpha}} \mathscr{L}\left\{\left(D_{w}^{2}+D_{z}^{2}\right) P_{n}-k r Q_{n}+k v_{n}\right\}\right],
\end{array} \quad n \geq 1 .
\end{aligned}
$$

\section{Applications}

In this section we present some application of LADM to solve biological population model. 
Example 6. Consider the following fractional order biological model [21]:

$$
\begin{aligned}
D_{t}^{\alpha} v(w, z, t)= & \left(D_{w}^{2}+D_{z}^{2}\right) v(w, z, t)-r v^{2}(w, z, t) \\
& +v(w, z, t)
\end{aligned}
$$

with given initial condition

$$
v(w, z, 0)=\exp \left(\frac{1}{2} \sqrt{\frac{r}{2}}(w+z)\right) .
$$

Upon using the proposed method on (23) and comparing terms of both sides and then taking Laplace inverse transform, we get

$$
\begin{gathered}
v_{0}(w, z, t)=\exp \left(\frac{1}{2} \sqrt{\frac{r}{2}}(w+z)\right) \\
\mathscr{L}\left\{v_{1}(w, z, t)\right\}=\frac{1}{s^{\alpha}} \mathscr{L}\left\{\left(D_{w}^{2}+D_{z}^{2}-r\right) P_{0}+v_{0}\right\} \\
v_{1}(w, z, t)=\exp \left(\frac{1}{2} \sqrt{\frac{r}{2}}(w+z)\right) \frac{t^{\alpha}}{\Gamma(\alpha+1)}, \\
v_{2}(w, z, t)=\exp \left(\frac{1}{2} \sqrt{\frac{r}{2}}(w+z)\right) \frac{t^{2 \alpha}}{\Gamma(2 \alpha+1)}, \\
v_{3}(w, z, t)=\exp \left(\frac{1}{2} \sqrt{\frac{r}{2}}(w+z)\right) \frac{t^{3 \alpha}}{\Gamma(3 \alpha+1)}
\end{gathered}
$$

and so on.

The series solution is provided as

$$
\begin{aligned}
v= & \exp \left(\frac{1}{2} \sqrt{\frac{r}{2}}(w+z)\right)+\exp \left(\frac{1}{2} \sqrt{\frac{r}{2}}(w+z)\right) \\
& \cdot \frac{t^{\alpha}}{\Gamma(\alpha+1)}+\exp \left(\frac{1}{2} \sqrt{\frac{r}{2}}(w+z)\right) \frac{t^{2 \alpha}}{\Gamma(2 \alpha+1)} \\
& +\cdots \\
v= & \exp \left(\frac{1}{2} \sqrt{\frac{r}{2}}(w+z)\right) \\
& \cdot\left[1+\frac{t^{\alpha}}{\Gamma(\alpha+1)}+\frac{t^{2 \alpha}}{\Gamma(\alpha+1)}+\frac{t^{3 \alpha}}{\Gamma(3 \alpha+1)}+\cdots\right] .
\end{aligned}
$$

In closed form, the solution is given by

$$
v(w, z, t)=\exp \left(\frac{1}{2} \sqrt{\frac{r}{2}}(w+z)\right) E_{\alpha}\left(t^{\alpha}\right)
$$

which is the exact solution. Putting $\alpha=1$ in (27), we get solution as

$$
v(w, z, t)=\exp \left(\frac{1}{2} \sqrt{\frac{r}{2}}(w+z)+t\right) .
$$

This is the classical solution.
Example 7. Consider the following fractional order biological model [21]:

$$
D_{t}^{\alpha} v(w, z, t)=\left(D_{w}^{2}+D_{z}^{2}\right) v^{2}(w, z, t)+v(w, z, t)
$$

with given initial condition

$$
v(w, z, 0)=\sqrt{\sin w \sinh z} .
$$

Applying Laplace transform on both sides, we have

$$
\begin{aligned}
& \mathscr{L}\left\{D_{t}^{2} v\right\}=\mathscr{L}\left\{\left(D_{2}^{w}+D_{2}^{z}\right)+v_{n}\right\} \\
& s^{\alpha} \mathscr{L}\{v(w, z, t)\}-s^{\alpha-1} v(w, z, 0) \\
& \quad=\mathscr{L}\left\{\left(D_{w}^{2}+D_{z}^{2}\right) v_{n}^{2}(w, z, t)+v_{n}(w, z, t)\right\} \\
& \mathscr{L}\left\{v_{n}(w, z, t)\right\}=\frac{1}{s} v(w, z, 0) \\
& \quad+\frac{1}{s^{\alpha}} \mathscr{L}\left\{\left(D_{w}^{2}+D_{z}^{2}\right) v_{n}^{2}+v_{n}\right\} \\
& \mathscr{L}\left\{v_{0}(w, z, t)\right\}=\frac{1}{s} \sqrt{\sin w \sinh z} \\
& \mathscr{L}\left\{v_{1}(w, z, t)\right\} \\
& \quad=\frac{1}{s^{\alpha}} \mathscr{L}\left\{\left(D_{w}^{2}+D_{z}^{2}\right) v_{0}^{2}(w, z, t)+v_{0}(w, z, t)\right\} \\
& \mathscr{L}\left\{v_{2}(w, z, t)\right\} \\
& \quad=\frac{1}{s^{\alpha}} \mathscr{L}\left\{\left(D_{w}^{2}+D_{z}^{2}\right) v_{1}^{2}(w, z, t)+v_{1}(w, z, t)\right\} \\
& \mathscr{L}\left\{v_{3}(w, z, t)\right\} \\
& \quad=\frac{1}{s^{\alpha}} \mathscr{L}\left\{\left(D_{w}^{2}+D_{z}^{2}\right) v_{2}^{2}(w, z, t)+v_{2}(w, z, t)\right\} \\
& \vdots
\end{aligned}
$$

After using Laplace inverse transform on both sides of (31), we have

$$
v_{0}(w, z, t)=\sqrt{\sin w \sinh z}
$$

$$
\begin{aligned}
& v_{1}(w, z, t)=\sqrt{\sin w \sinh z} \frac{t^{\alpha}}{\Gamma(\alpha+1)} \\
& v_{2}(w, z, t)=\sqrt{\sin w \sinh z} \frac{t^{2 \alpha}}{\Gamma(2 \alpha+1)} \\
& v_{3}(w, z, t)=\sqrt{\sin w \sinh z} \frac{t^{3 \alpha}}{\Gamma(3 \alpha+1)}
\end{aligned}
$$


and so no. Now the series solution of problem (29) is given by

$$
\begin{aligned}
& v(w, z, t)=v_{0}(w, z, t)+v_{1}(w, z, t)+v_{2}(w, z, t) \\
& +\cdots \\
& v(w, z, t)=\sqrt{\sin w \sinh z}+\sqrt{\sin w \sinh z} \frac{t^{\alpha}}{\Gamma(\alpha+1)} \\
& +\sqrt{\sin w \sinh z} \frac{t^{2 \alpha}}{\Gamma(2 \alpha+1)}+\sqrt{\sin w \sinh z} \\
& \quad \cdot \frac{t^{3 \alpha}}{\Gamma(3 \alpha+1)}+\cdots \\
& v(w, z, t)=\sqrt{\sin w \sinh z}\left[1+\frac{t^{\alpha}}{\Gamma(\alpha+1)}+\frac{t^{2 \alpha}}{\Gamma(\alpha+1)}\right. \\
& \left.\quad+\frac{t^{3 \alpha}}{\Gamma(3 \alpha+1)}+\cdots\right] \cdot
\end{aligned}
$$

The closed form is given by

$$
v(w, z, t)=\sqrt{\sin w \sinh z} E_{\alpha}\left(t^{\alpha}\right) .
$$

Considering $\alpha=1$, we get classical solution as

$$
v(w, z, t)=\sqrt{\sin w \sinh z} e^{t}
$$

Example 8. Consider the following fractional order biological model.

$$
D_{t}^{\alpha} v(w, z, t)=\left(D_{w}^{2}+D_{z}^{2}\right) v(w, z, t)+k v(w, z, t),
$$

corresponding to the initial condition

$$
v(w, z, 0)=\sqrt{w z}
$$

Applying Laplace transform on both sides, we have

$$
\begin{aligned}
\mathscr{L} & \left\{D_{t}^{2} v(w, z, t)\right\} \\
& =\mathscr{L}\left\{\left(D_{2}^{w}+D_{2}^{z}\right) v_{n}^{2}(w, z, t)+k v_{n}(w, z, t)\right\} \\
s^{\alpha} & \mathscr{L}\{v(w, z, t)\}-s^{\alpha-1} v(w, z, 0) \\
& =\mathscr{L}\left\{\left(D_{w}^{2}+D_{z}^{2}\right) v_{n}^{2}(w, z, t)+v_{n}(w, z, t)\right\}
\end{aligned}
$$

$$
\begin{aligned}
& \mathscr{L}\left\{v_{n}(w, z, t)\right\}=\frac{1}{s} v(w, z, 0) \\
& +\frac{1}{s^{\alpha}} \mathscr{L}\left\{\left(D_{w}^{2}+D_{z}^{2}\right) v_{n}^{2}(w, z, t)+v_{n}(w, z, t)\right\} \\
& \mathscr{L}\left\{v_{0}(w, z, t)\right\}=\frac{1}{s} \sqrt{w z} \\
& \mathscr{L}\left\{v_{1}(w, z, t)\right\} \\
& \quad=k \frac{1}{s^{\alpha}} \mathscr{L}\left\{\left(D_{w}^{2}+D_{z}^{2}\right) v_{0}^{2}(w, z, t)+v_{0}(w, z, t)\right\} \\
& \mathscr{L}\left\{v_{2}(w, z, t)\right\} \\
& \quad=k \frac{1}{s^{\alpha}} \mathscr{L}\left\{\left(D_{w}^{2}+D_{z}^{2}\right) v_{1}^{2}(w, z, t)+v_{1}(w, z, t)\right\} \\
& \mathscr{L}\left\{v_{3}(w, z, t)\right\} \\
& \quad=k \frac{1}{s^{\alpha}} \mathscr{L}\left\{\left(D_{w}^{2}+D_{z}^{2}\right) v_{2}^{2}(w, z, t)+v_{2}(w, z, t)\right\} \\
& \vdots
\end{aligned}
$$

After using Laplace transform on both sides, we have

$$
\begin{gathered}
v_{0}(w, z, t)=\sqrt{w z} \\
v_{1}(w, z, t)=k \sqrt{w z} \frac{t^{\alpha}}{\Gamma(\alpha+1)} \\
v_{2}(w, z, t)=k^{2} \sqrt{w z} \frac{t^{2 \alpha}}{\Gamma(2 \alpha+1)} \\
v_{3}(w, z, t)=k^{3} \sqrt{w z} \frac{t^{3 \alpha}}{\Gamma(3 \alpha+1)}
\end{gathered}
$$

and so on. The series solution of problem (36) is given by

$$
\begin{aligned}
& v(w, z, t)=v_{0}(w, z, t)+v_{1}(w, z, t)+v_{2}(w, z, t) \\
& \quad+\cdots
\end{aligned}
$$

$$
\begin{gathered}
v(w, z, t)=\sqrt{w z}+k \sqrt{w z} \frac{t^{\alpha}}{\Gamma(\alpha+1)}+k^{2} \sqrt{w z} \\
\cdot \frac{t^{2 \alpha}}{\Gamma(2 \alpha+1)}+h^{3} \sqrt{w z} \frac{t^{3 \alpha}}{\Gamma(3 \alpha+1)}+\cdots
\end{gathered}
$$

$$
\begin{aligned}
& v(w, z, t)=\sqrt{w z}\left[1+k \frac{t^{\alpha}}{\Gamma(\alpha+1)}+k^{2} \frac{t^{2 \alpha}}{\Gamma(\alpha+1)}\right. \\
& \left.+k^{3} \frac{t^{3 \alpha}}{\Gamma(3 \alpha+1)}+\cdots\right] .
\end{aligned}
$$

Hence closed form of the solution is given by

$$
v(w, z, t)=\sqrt{w z} E_{\alpha}\left(k t^{\alpha}\right) .
$$




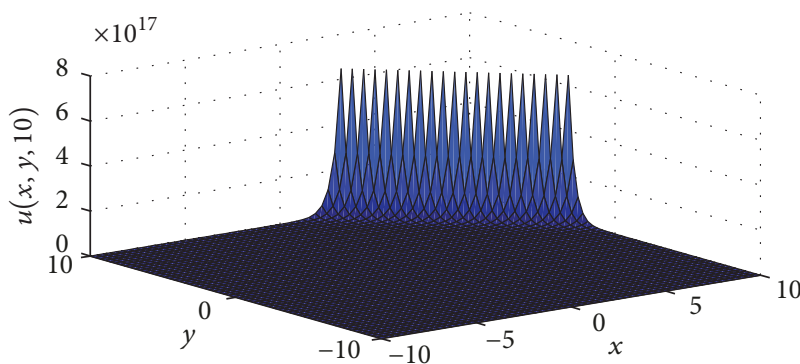

$\alpha=1.0$

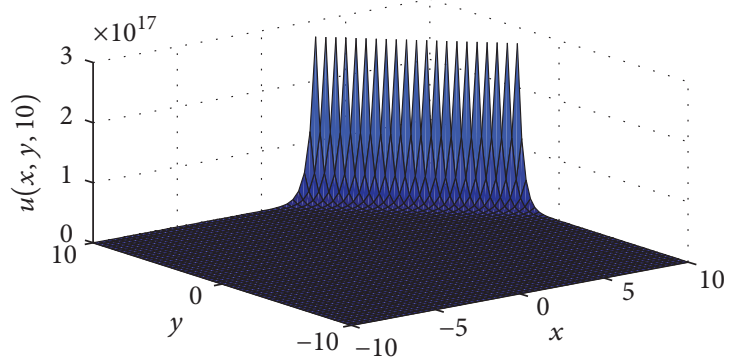

$\alpha=0.95$ (a)

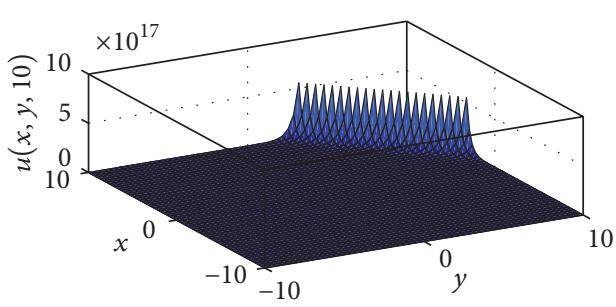

$\alpha=0.85$

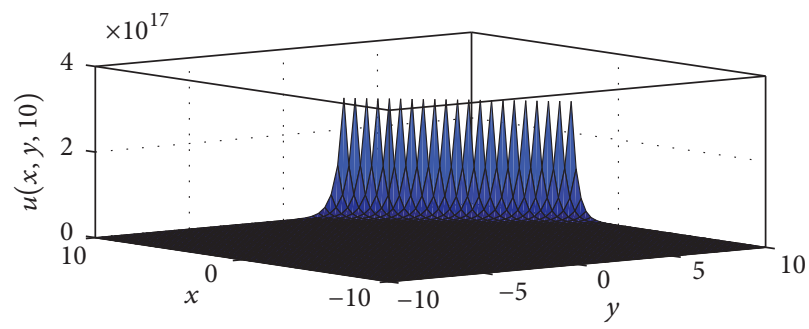

$\alpha=0.75$

(c)

(d)

FIgURE 1: Numerical plots of approximate solutions of Example 6 at various fractional orders and using $r=50, t=10$.

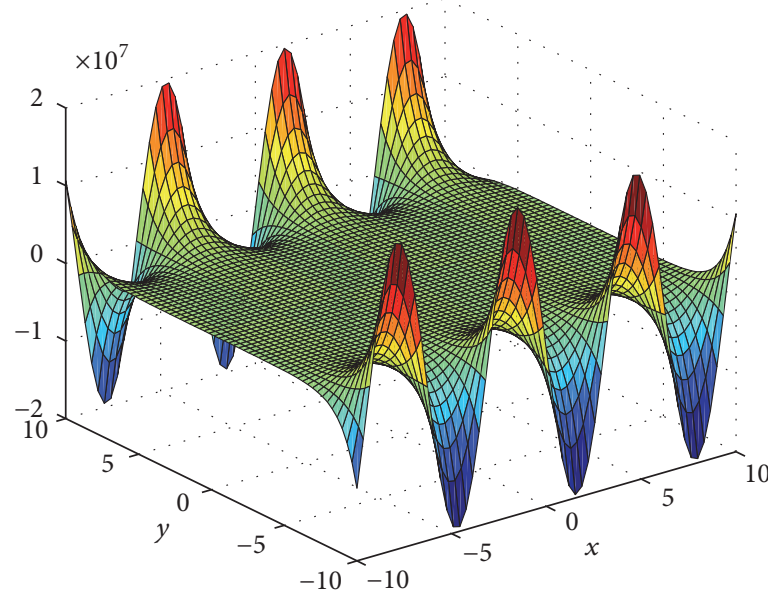

$\alpha=0.75$

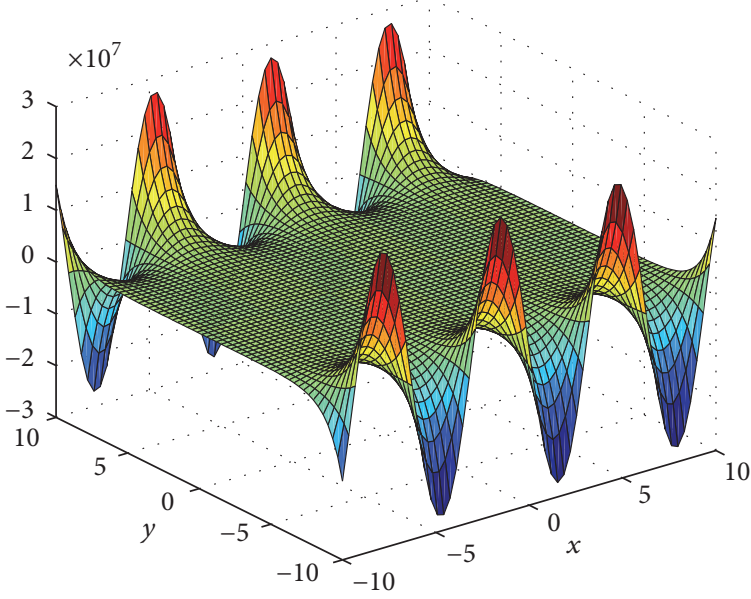

$\alpha=0.85$

(a)

(b)

Figure 2: Numerical plots of approximate solutions of Example 7 at various fractional orders and using $t=10$.

Considering $\alpha=1$, we get classical solution as

$$
v(w, z, t)=\sqrt{w z} e^{t} .
$$

\section{Conclusion and Discussion}

In this article, the (LADM) has been applied successfully to obtain the exact solution of the generalized fractional order biological model with given initial conditions. From the numerical plots given in Figures 1, 2, and 3 of the considered examples, we see that the procedure is efficient to obtain approximate or exact solutions to fractional order partial differential equations corresponding to various fractional order. In the current situation we have come across the exact solution for the corresponding nonlinear FPDEs.

In the 1980s George Adomian formulated a novel powerful scheme for solving nonlinear functional equations. Afterward, in the literature this method was known as Adomian 


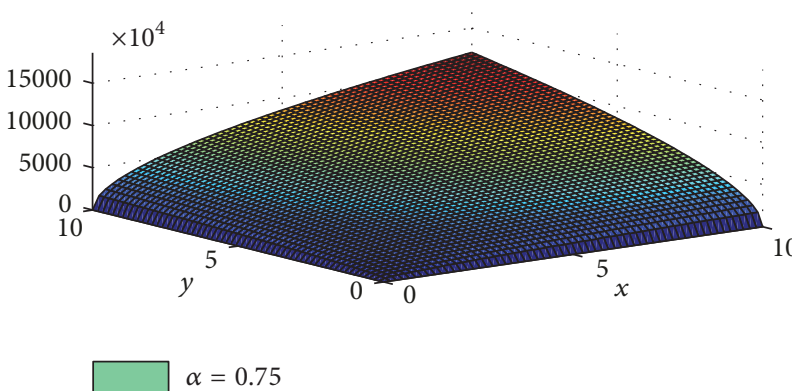

(a)

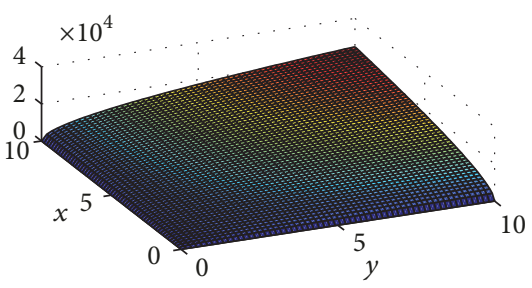

$\alpha=0.95$

(c)

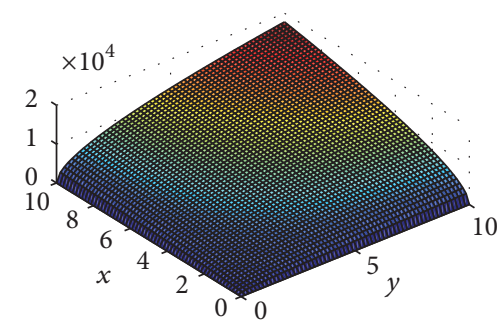

$\alpha=0.85$

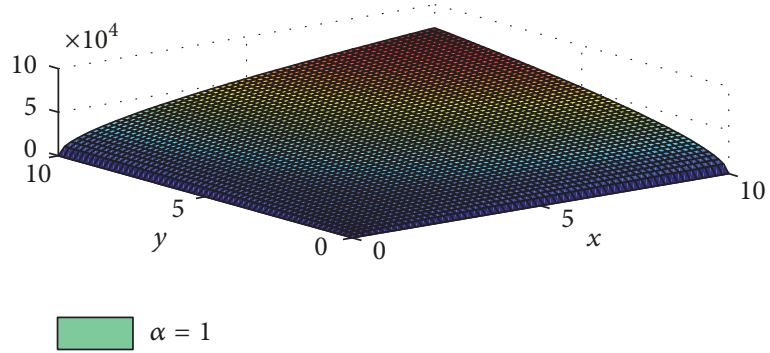

(d)

FIGURE 3: Numerical plots of approximate solutions of Example 8 at various fractional orders and using $k=0.1, t=10$.

decomposition method (ADM). The technique is based on the splitting of a system of differential equations solutions in the form of series of functions. Every term of the associated series is obtained from a polynomial generated by expansion of an analytic function into a power series. This is an effective tool for solution of systems of differential equations appearing in physical problems. Over the last 20 years, the Adomian decomposition approach has been applied to obtain formal solutions to a wide class of stochastic and deterministic problems involving algebraic, differential, integrodifferential, differential delay, integral, and partial differential equations. As compared to other existing numerical schemes, the main advantage of this method is that it does not require perturbation or liberalization for exploring the dynamical behavior of complex dynamical systems. The LADM has done extensive work to provide analytical solution of nonlinear equations as well as solving differential equations of fractional order.

Further in this paper, the obtained results have been compared with the analytical solution and with the results obtained by Adomian decomposition method [20] and homotopy analysis method. In [20], the authors show that ADM generally does not converge, when the method is applied to highly nonlinear differential equation. Our proposed method is better than HAM, HPM, and VIM, because it needs no parameter terms to form decomposition equation, no perturbation as needed in the mention methods. Our proposed method is simple and does not require or waste extra memory like Tau-collocation method. Further from direct ADM, our method is better as we applied Laplace transform and then decompose the nonlinear terms in terms of Adomian polynomials, while in Adomian decomposition particular integral is involved, which often creates difficulties in computation. The main advantage of this technique is that it avoids complex transformations like index reductions and leads to a simple general algorithm. Secondly, it reduces the computational work by solving only linear algebraic systems. From this method, we obtained a simple way to control the convergence region of the series solution by using a proper value of parameters. The results show that LADM is very efficient, powerful method to find the analytical solution of nonlinear differential equations.

\section{Conflicts of Interest}

There are no conflicts of interest regarding this paper.

\section{Authors' Contributions}

All authors equally contributed to this paper and approved the final version.

\section{Acknowledgments}

This work has been supported by the National Natural Science Foundation of China (11571378).

\section{References}

[1] A. A. Kilbas, O. I. Marichev, and S. G. Samko, Fractional Integrals and Derivatives, Theory and Applications, Gordon and Breach, Yverdon, Switzerland, 1993.

[2] K. S. Miller and B. Ross, An Introduction to the Fractional Calculus and Fractional Differential Equations, A Wiley-Interscience Publication, John Wiley \& Sons, New York, NY, USA, 1993. 
[3] I. Podlubny, Fractional Differential Equations, vol. 198 of Mathematics in Science and Engineering, Academic Press, San Diego, Calif, USA, 1999.

[4] F. Haq, K. Shah, G. Rahman, and M. Shahzad, "Existence and uniqueness of positive solution to boundary value problem of fractional," Sindh University Research Journal, vol. 48, no. 2, pp. 451-456, 2016.

[5] A. A. Kilbas, H. M. Srivastava, and J. J. Trujillo, Theory and Applications of Fractional Differential Equations, New York, NY, USA, Elsevier, 2006.

[6] A. Atangana and D. Baleanu, "New fractional derivatives with non-local and non-singular kernel: Theory and application to heat transfer model," Thermal Science, vol. 20, no. 2, pp. 763769, 2016.

[7] D. D. Ganji, “The application of He's homotopy perturbation method to nonlinear equations arising in heat transfer," Physics Letters A, vol. 355, no. 4-5, pp. 337-341, 2006.

[8] F. Haq, K. Shah, G. ur Rahman, and M. Shahzad, "Numerical solution of fractional order smoking model via laplace Adomian decomposition method," Alexandria Engineering Journal, 2016.

[9] F. Haq, K. Shah, G.-U. Rahman, and M. Shahzad, "Numerical analysis of fractional order model of HIV-1 infection of CD4+ T-cells," Computational Methods for Differential Equations, vol. 5, no. 1, pp. 1-11, 2017.

[10] J. Biazar, M. Gholami Porshokuhi, and B. Ghanbari, "Extracting a general iterative method from an Adomian decomposition method and comparing it to the variational iteration method," Computers \& Mathematics with Applications, vol. 59, no. 2, pp. 622-628, 2010.

[11] H. Kheiri, N. Alipour, and R. Dehghani, "Homotopy analysis and homotopy Pade methods for the modified BurgersKorteweg-de Vries and the Newell-Whitehead equations," Mathematical Sciences Quarterly Journal, vol. 5, no. 1, pp. 33-50, 2011.

[12] Y. Khan and N. Faraz, "A new approach to differential difference equations," Journal of Advanced Research in Differential Equations, vol. 2, pp. 1-12, 2010.

[13] M. Khan and M. Hussain, "Application of Laplace decomposition method on semi-infinite domain," Numerical Algorithms, vol. 56, no. 2, pp. 211-218, 2011.

[14] Y. Khan and Q. Wu, "Homotopy perturbation transform method for nonlinear equations using He's polynomials," Computers \& Mathematics with Applications, vol. 61, no. 8, pp. 19631967, 2011.

[15] M. A. Gondal, A. S. Arife, M. Khan, and I. Hussain, "An efficient numerical method for solving linear and nonlinear partial differential equations by combining homotopy analysis and transform method," World Applied Sciences Journal, vol. 14, no. 12, pp. 1786-1791, 2011.

[16] M. Khan, M. A. Gondal, I. Hussain, and S. K. Vanani, "A new comparative study between homotopy analysis transform method and homotopy perturbation transform method on a semi infinite domain," Mathematical and Computer Modelling, vol. 55, no. 3-4, pp. 1143-1150, 2012.

[17] D. Baleanu, O. Defterli, and O. P. Agrawal, "A central difference numerical scheme for fractional optimal control problems," Journal of Vibration and Control, vol. 15, no. 4, pp. 583-597, 2009.

[18] A. K. Golmankhaneh, A. K. Golmankhaneh, and D. Baleanu, "Homotopy perturbation method for solving a system of Schrödinger-Korteweg-de Vries equations," Romanian Reports in Physics, vol. 63, no. 3, pp. 609-623, 2011.
[19] D. Kumar, J. Singh, and Sushela, "Application of homotopy analysis transform method to fractional biological population model," Romanian Reports Physics, vol. 65, no. 1, pp. 63-75, 2013.

[20] A. A. Arafa, S. Z. Rida, and H. Mohamed, "Homotopy analysis method for solving biological population model," Communications in Theoretical Physics, vol. 56, no. 5, pp. 797-800, 2011.

[21] A. M. El-Sayed, S. Z. Rida, and A. A. Arafa, "Exact solutions of fractional-order biological population model," Communications in Theoretical Physics, vol. 52, no. 6, pp. 992-996, 2009.

[22] M. Caputo, Elasticita e Dissipazione, Zani-Chelli, Bologna, Italy, 1969.

[23] G. Rudolf and F. Mainardi, Fractals and Fractional Calculus in Continuum Mechanics, Springer, Vienna, Austria, 1997. 


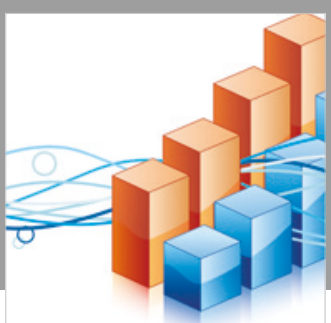

Advances in

Operations Research

\section{-n-m}
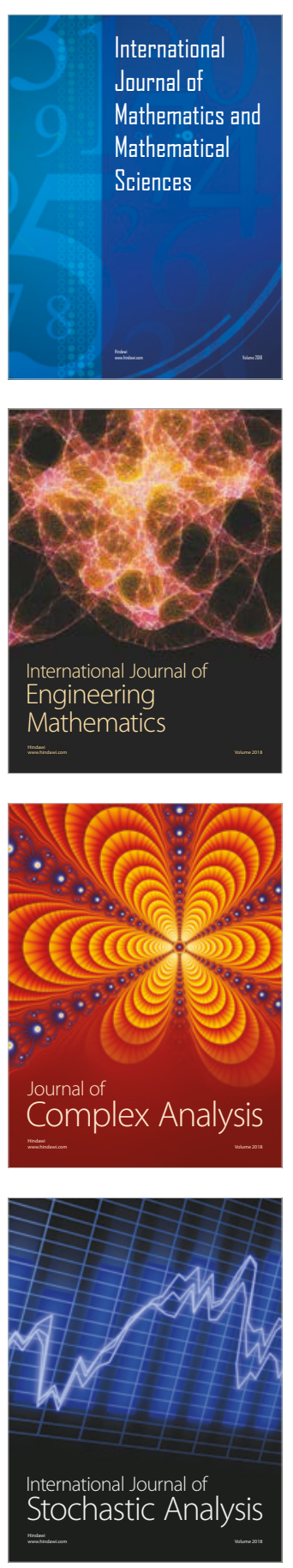
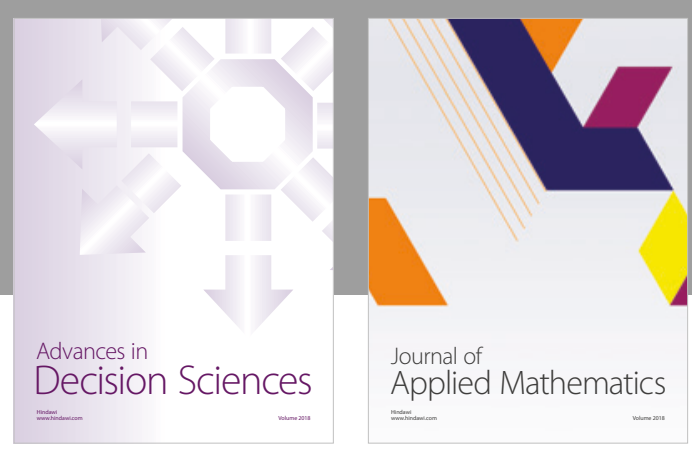

Journal of

Applied Mathematics
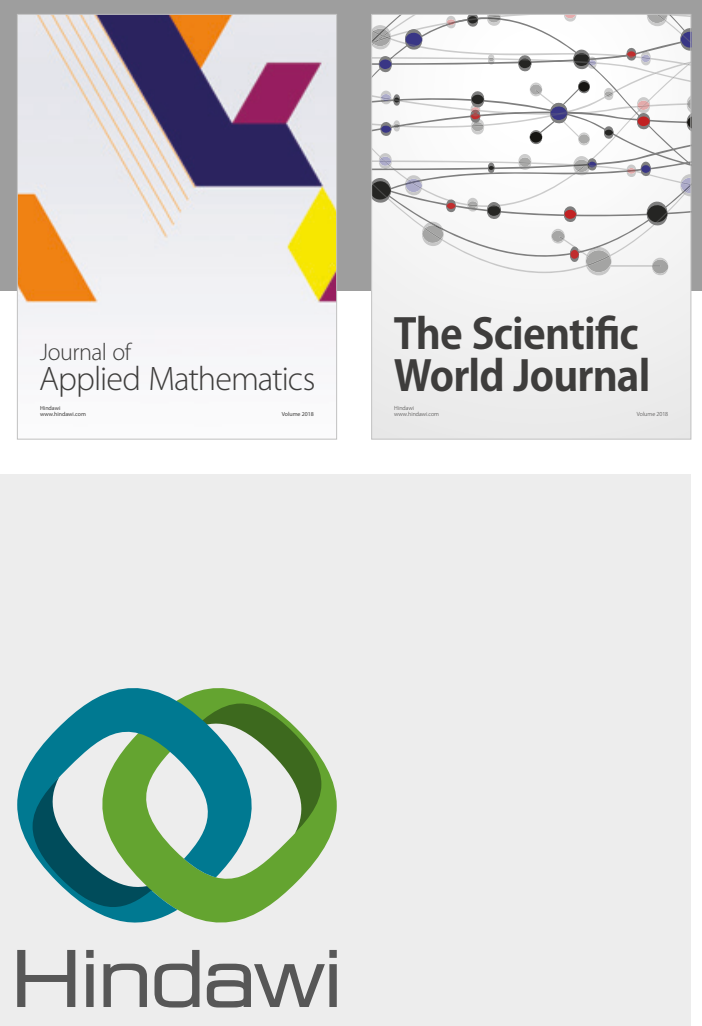

Submit your manuscripts at

www.hindawi.com

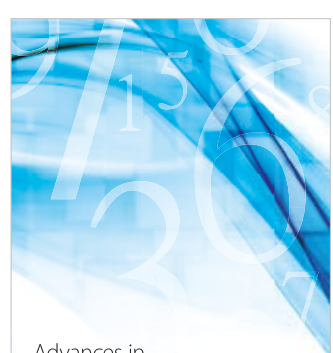

Advances in
Numerical Analysis
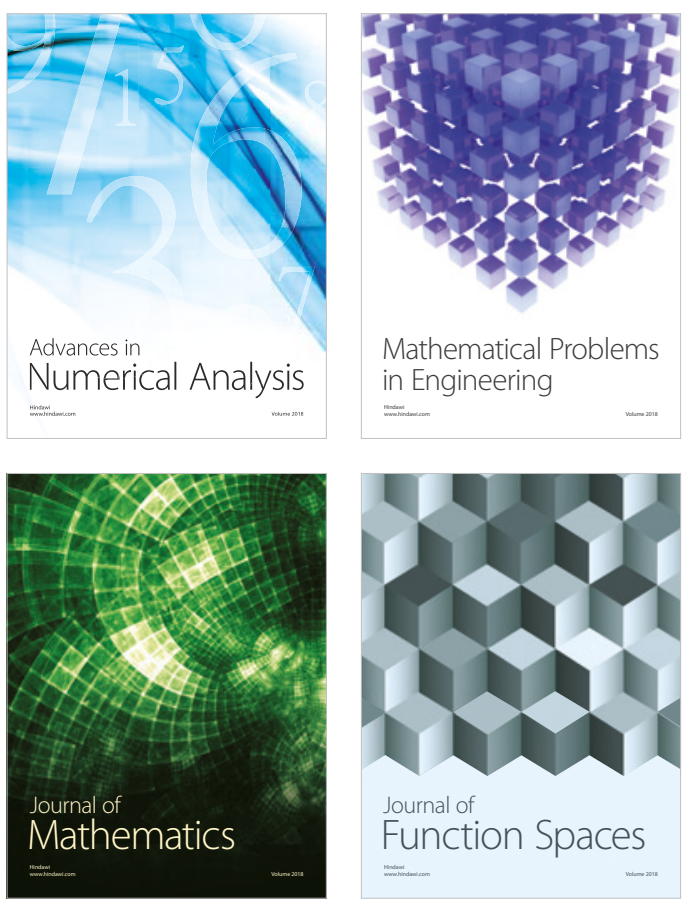

Mathematical Problems in Engineering

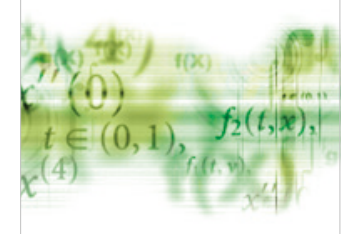

International Journal of

Differential Equations

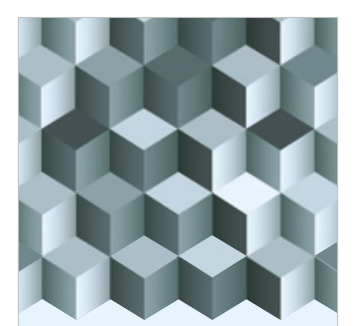

Journal of

Function Spaces
The Scientific

World Journal

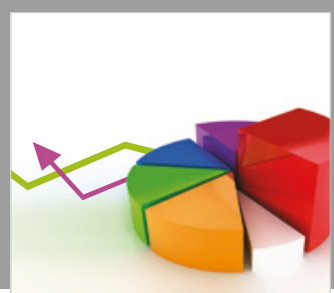

Journal of

Probability and Statistics
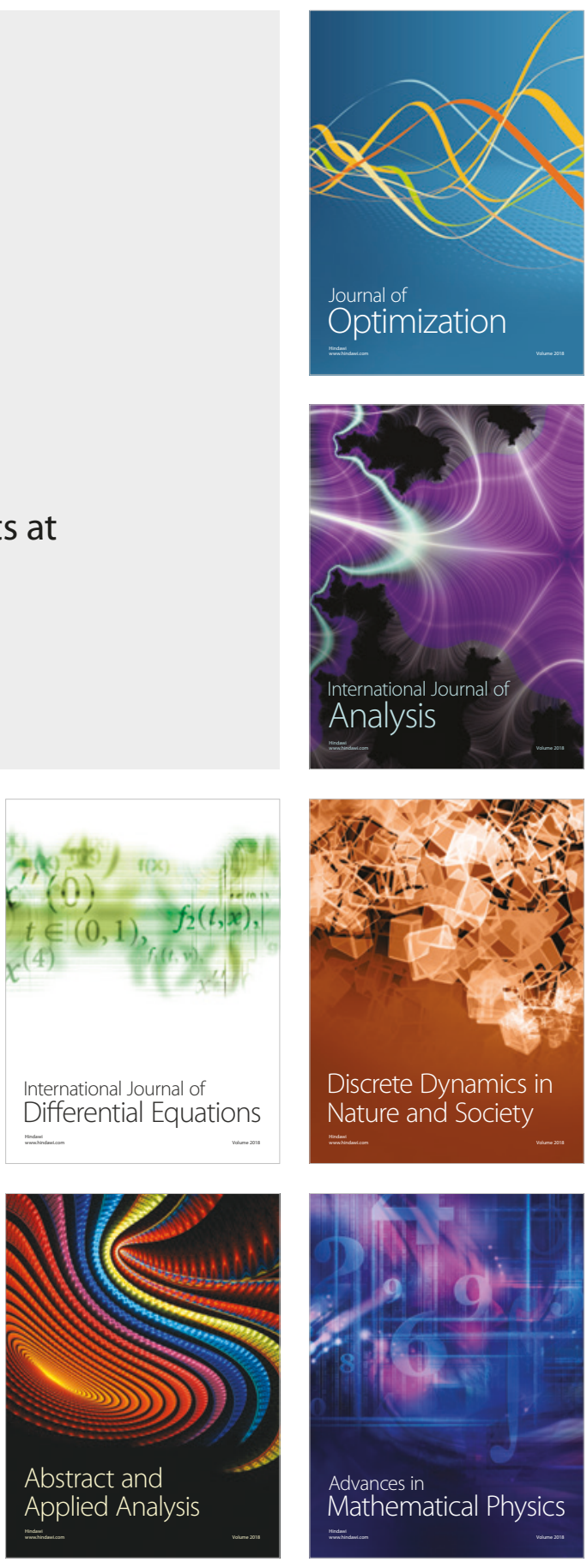